OPEN ACCESS

Edited by:

Haijin Mou,

Ocean University of China, China

Reviewed by: Han Sun,

Ocean University of China, China

Hongli Cui,

Shanxi Agricultural University, China

*Correspondence:

Lijun Li

ljil@jmu.edu.cn

Specialty section:

This article was submitted to

Bioprocess Engineering,

a section of the journal

Frontiers in Bioengineering and

Biotechnology

Received: 10 November 2021

Accepted: 29 December 2021

Published: 17 January 2022

Citation:

$L i Z$, Yang $H$, Zheng $C, D u X, N i H$,

He N, Yang L, You L, Zhu Y and Li L (2022) Effectively Improve the

Astaxanthin Production by Combined

Additives Regulating Different

Metabolic Nodes in

Phaffia rhodozyma.

Front. Bioeng. Biotechnol. 9:812309.

doi: 10.3389/fbioe.2021.812309

\section{Effectively Improve the Astaxanthin Production by Combined Additives Regulating Different Metabolic Nodes in Phaffia rhodozyma}

\author{
Zhipeng $\mathrm{Li}^{1,2,3}$, Haoyi Yang ${ }^{1,2,3}$, Chenhua Zheng ${ }^{1,2,3}$, Xiping $\mathrm{Du}^{1,2,3}, \mathrm{Hui} \mathrm{Ni}^{1,2,3}, \mathrm{Ning} \mathrm{He}^{4}$, \\ Liang Yang ${ }^{1,2,3}$, Li You ${ }^{1,2,3}$, Yanbing Zhu ${ }^{1,2,3}$ and Lijun $\mathrm{Li}^{1,2,3 *}$ \\ ${ }^{1}$ College of Food and Biology Engineering, Jimei University, Xiamen, China, ${ }^{2}$ Fujian Provincial Key Laboratory of Food \\ Microbiology and Enzyme Engineering Technology, Xiamen, China, ${ }^{3}$ Research Center of Food Biotechnology of Xiamen City, \\ Xiamen, China, ${ }^{4}$ Department of Chemical and Biochemical Engineering, College of Chemistry and Chemical Engineering, Xiamen \\ University, Xiamen, China
}

Astaxanthin is an important natural resource that is widely found in marine environments. Metabolic regulation is an effective method for improving astaxanthin production in Phaffia rhodozyma. Most studies have focused on single regulators, which have limited effects. In this study, 16 metabolic regulators were screened to improve astaxanthin production in high-yield and wild-type strains. Fluconazol and glutamic acid increased astaxanthin volumetric yield in MVP14 by 25.8 and $30.9 \%$, respectively, while ethanol increased astaxanthin volumetric yield in DSM626, 29.3\%. Furthermore, six additives that inhibit the competing pathways and promote the main pathway for astaxanthin synthesis were selected for combination treatment. We found that the optimal combination was penicillin, ethanol, triclosan, and fluconazol, which increased astaxanthin cell yield by $51 \%$. Therefore, we suggest that simultaneously promoting the master pathways (mevalonate) and inhibiting competing pathways (fatty acid synthesis and ergosterol) is the best strategy to improve astaxanthin cell yield. Moreover, regulators of the biomass pathway should be avoided to improve cell yield. This study provides a technical basis for the utilisation of astaxanthin in P. rhodozyma.

Keywords: phaffia rhodozyma, astaxanthin, metabolic pathways, metabolic regulators, combined additives

After adding penicillin, ethanol, triclosan and fluconazol, the changes happened in the metabolic pathways of $P$. rhodozyma cells. Red represents up-regulation of the substance or pathway, and green represents down-regulation.

\section{INTRODUCTION}

Astaxanthin (3,3-dihydroxy-beta,beta-carotene-4,4'-dione) is a ketocarotenoid with antioxidant, antiinflammatory, anti-aging, anti-tumour, and immune-enhancing properties (Mimoun-Benarroch et al., 2018; Lai et al., 2021; Zhou et al., 2021). It is widely present in marine environments and plays an important role in the survival of various marine organisms (Schmidt et al., 2011). The main natural sources of astaxanthin are Phaffia rhodozyma and Haematococcus pluvialis (Schmidt et al., 2011). P. rhodozyma, a basidiomycete, is an important biological source of astaxanthin (Liu et al., 2021; Torres-Haro et al., 2021). 


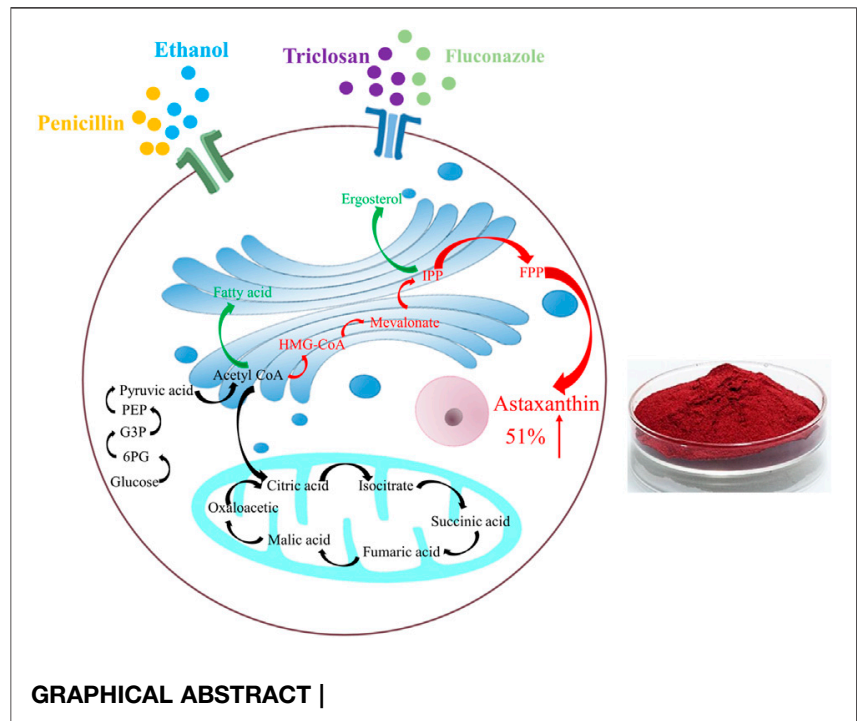

Compared with other strains, the fermentation of astaxanthin by $P$. rhodozyma offers many advantages, such as simple culture requirements and ease of production (Stoklosa et al., 2019). The astaxanthin yield of wild $P$. rhodozyma is low at only $0.17-1.73 \mathrm{mg} / \mathrm{g}$ dry cell (Domínguez-Bocanegra, 2007; Batghare et al., 2018), and cannot meet industrial production requirements (Kothari et al., 2019).

Metabolic regulation is an important method to improve microbial productivity (Lai et al., 2021). The production of astaxanthin in $P$. rhodozyma can be affected by many complex metabolic pathways, such as the hexose monophosphate (HMP) pathway, EmbdenMeyerhof-Parnas (EMP) pathway, mevalonate (MVA) pathway, tricarboxylic acid (TCA) cycle, fatty acid synthesis (FAS) pathway, and ergosterol synthesis pathway (Pan et al., 2020; Wan et al., 2021; Zhang et al., 2021). Among these pathways, HMP, EMP, and MVA pathways are necessary in the synthesis of astaxanthin (Lan et al., 2002; Wang et al., 2012; Wushensky et al., 2018), whereas TCA, FAS, and ergosterol synthesis pathways are competing pathways (Sanglard et al., 1998; Cheng et al., 2016; Wang, L. et al., 2019). The production of carotenoids, including astaxanthin, can be increased by adding metabolic regulators which affect these metabolic pathways. Gu et al. (1997) increased the carotenoid production in P. rhodozyma by $0.84 \mathrm{mg} / \mathrm{g}$ by adding $0.2 \%$ ethanol to the medium. Miao et al. (2011) increased astaxanthin production in P. rhodozyma by 0.01 and $0.2 \mathrm{mg} / \mathrm{g}$ in $96 \mathrm{~h}$ by adding triclosan and fluconazol, respectively. Chen et al. (2021) reported that pyruvate can increase astaxanthin content in Chromochloris zofingiensis by $25 \%$. It can be seen that single modulator is effective but its impact is very limited.

Therefore, systematic screening of single regulators could help analyse their regulatory functions and effects on metabolic pathways. Previous studies have shown that inhibiting competing pathways can remarkably increase the yield of target products. Lee et al. (2018) reported that the elimination of L-valine and L-isoleucine can enhance isobutanol production in Saccharomyces cerevisiae. Similarly, the promotion and regulation of synthetic pathways can also achieve positive results; for example, light can promote carotenoid accumulation (Sun et al., 2019). Therefore, we speculated that biosynthetic and competing metabolic pathways could be regulated simultaneously to achieve a more optimal effect.

In this study, we explored the effects of 15 related additives on the above mentioned six pathways. These additives are known to exert promotive or competitive effects on astaxanthin production. We further optimised a combination of these additives. Furthermore, the effects of different metabolic pathways on astaxanthin synthesis were systematically analysed to investigate the mechanism of astaxanthin synthesis. This study could provide technical support to improve the astaxanthin industry and provide a theoretical basis for the study of astaxanthin metabolism.

\section{MATERIAL AND METHODS}

\section{Strains and Culture Conditions}

P. rhodozyma JMU-MVP14 was preserved by Fujian Provincial Key Laboratory of Food Microbiology and Enzyme Engineering Technology, Jimei University. The astaxanthin-overproducing mutant $P$. rhodozyma JMU-MVP14 was established through ethyl methyl sulfonate mutagenesis from JMU-VDL668. P. rhodozyma DSM5626 (ATCC 24202) was purchased from the German Collection of Microorganisms and Cell Cultures (Germany).

Two generations of cultivation were performed in yeast extract-peptone-dextrose (YPD) medium. Then, each strain was fermented in another YPD medium. The YPD medium, which was composed of $10 \mathrm{~g} / \mathrm{L}$ yeast powder, $20 \mathrm{~g} / \mathrm{L}$ peptone, and $20 \mathrm{~g} / \mathrm{L}$ glucose and had a $\mathrm{pH}$ of 6.0 , was sterilized at $121^{\circ} \mathrm{C}$ for $30 \mathrm{~min}$. Add $30 \mathrm{ml}$ of fresh medium to the $250 \mathrm{ml}$ Erlenmeyer flask and inoculate with $3 \%$ of the inoculum. The first-generation cultured $48 \mathrm{~h}$, the second-generation cultured $24 \mathrm{~h}$. The culture temperature was $22^{\circ} \mathrm{C}$, and the rotational speed of shaker was $200 \mathrm{r} / \mathrm{min}$.

\section{Addition Method}

Different regulators, namely, pyruvic acid, glutamic acid, sodium fluoride, $a$-ketoglutarate, simvastatin, $\mathrm{N}$-methyl morpholine, malonic acid, nicotine, citric acid, sodium gluconate, and sodium phosphate, were added before sterilization. Ethanol was added during inoculation, penicillin was added at $36 \mathrm{~h}$ of fermentation, and fluconazol and triclosan were added according to different conditions. The concentrations and time of the regulations are shown in Table $\mathbf{1 .}$

The combination mode and concentrations of combined additives are shown in Table 2. Eighteen different combinations of additives include 15 combinations of two regulators, a combination of four regulators, and two combinations of five regulators.

\section{Determination of Carotenoid Content}

Dry weight method was used in the analysis. An appropriate volume of fermentation broth was centrifuged for $5 \mathrm{~min}$ at $4,000 \mathrm{rpm}$, and the yeast was washed twice with distilled water and dried to constant weight at $105^{\circ} \mathrm{C}$.

Astaxanthin in the samples was detected by spectrophotometry. DMSO method was used to break the walls (Sedmak et al., 1990; Ni et al., 2008). The fermentation broth $(1 \mathrm{ml})$ was collected and centrifuged, and the pellet was washed twice with distilled water and added with $2 \mathrm{ml}$ of DMSO 
TABLE 1 | Adding method of metabolic regulators.

\begin{tabular}{|c|c|c|c|c|}
\hline Metabolic regulators & Added concentration & Time of adding & Pathway & References \\
\hline Glutamic acid & $3 \mathrm{~g} / \mathrm{L}$ & Before sterilization & Promoting HMP & An, 2001; Lan et al., 2002 \\
\hline Sodium fluoride & $1.5 \mathrm{mg} / \mathrm{L}$ & Before sterilization & Promoting HMP & GumiŃska and Sterkowicz, 1975 \\
\hline Sodium phosphate & $1.5 \mathrm{mg} / \mathrm{L}$ & Before sterilization & Inhibiting HMP & Liu et al., 2022 \\
\hline Sodium gluconate & $1 \mathrm{~g} / \mathrm{L}$ & Before sterilization & Promoting EMP & Wushensky et al. (2018) \\
\hline Citric acid & $2 \mathrm{~g} / \mathrm{L}$ & Before sterilization & Inhibiting EMP & Li et al. (2012) \\
\hline Penicillin & $1 \mathrm{mg} / \mathrm{L}$ & 36th $\mathrm{h}$ of fermentation & Promoting MVA & Wang et al. (2012) \\
\hline Malonic acid & $0.5 \mathrm{mg} / \mathrm{L}$ & Before sterilization & Inhibiting TCA Cycle & Wang, L. et al. (2019) \\
\hline Pyruvic acid & $2 \mathrm{~g} / \mathrm{L}$ & Before sterilization & Promoting TCA Cycle & Chen et al. (2021) \\
\hline a-ketoglutaric acid & $0.5 \mathrm{~g} / \mathrm{L}$ & Before sterilization & Promoting Accumulation of Acetyl CoA & Li et al. (2012) \\
\hline Nicotine & $0.04 \%$ & Before sterilization & Inhibiting dehydrogenase Activity & Fazeli et al. (2009) \\
\hline Triclosan & 5 mg/L & 48th $\mathrm{h}$ of fermentation & Inhibiting FAS Pathway & Cheng et al. (2016) \\
\hline Fluconazol & $120 \mathrm{mg} / \mathrm{L}$ & 48th $\mathrm{h}$ of fermentation & Inhibiting Ergosterol Synthesis Pathway & Sanglard et al. (1998) \\
\hline Ethanol & $1 \mathrm{~g} / \mathrm{L}$ & $\mathrm{O} \mathrm{h}$ of fermentation & Promoting HMGR Enzyme Activity & Gu et al. (1997) \\
\hline Simvastatin & $0.02 \mathrm{~g} / \mathrm{L}$ & Before sterilization & Inhibiting HMGR Enzyme Activity & Susilowati et al. (2020) \\
\hline N-methyl morpholine & $0.40 \%$ & Before sterilization & Inhibiting HMGR & Bhosale, (2004) \\
\hline
\end{tabular}

TABLE 2 | Combined addition of metabolic regulators.

Metabolic regulators

Glutamic acid + sodium gluconate

Glutamic acid + citric acid

Glutamic acid + penicillin

Glutamic acid + ethanol

Glutamic acid + triclosan

Glutamic acid + fluconazol

Pyruvic acid + sodium gluconate

Pyruvic acid + citric acid

Pyruvic acid + penicillin

Pyruvic acid + ethanol

Pyruvic acid + triclosan

Pyruvic acid + fluconazol

Glutamic acid + pyruvic acid

Penicillin + ethanol

Penicillin + ethanol + triclosan + fluconazol

Glutamic acid + penicillin + ethanol + triclosan + fluconazol

Pyruvic acid + penicillin + ethanol + triclosan + fluconazol
Triclosan + fluconazol

Added concentration

$3 \mathrm{~g} / \mathrm{L}+1 \mathrm{~g} / \mathrm{L}$
$3 \mathrm{~g} / \mathrm{L}+2 \mathrm{~g} / \mathrm{L}$
$3 \mathrm{~g} / \mathrm{L}+1 \mathrm{mg} / \mathrm{L}$
$3 \mathrm{~g} / \mathrm{L}+1 \mathrm{~g} / \mathrm{L}$
$3 \mathrm{~g} / \mathrm{L}+5 \mathrm{mg} / \mathrm{L}$
$3 \mathrm{~g} / \mathrm{L}+120 \mathrm{mg} / \mathrm{L}$
$2 \mathrm{~g} / \mathrm{L}+1 \mathrm{~g} / \mathrm{L}$
$2 \mathrm{~g} / \mathrm{L}+2 \mathrm{~g} / \mathrm{L}$
$2 \mathrm{~g} / \mathrm{L}+1 \mathrm{mg} / \mathrm{L}$
$2 \mathrm{~g} / \mathrm{L}+1 \mathrm{~g} / \mathrm{L}$
$2 \mathrm{~g} / \mathrm{L}+5 \mathrm{mg} / \mathrm{L}$
$2 \mathrm{~g} / \mathrm{L}+120 \mathrm{mg} / \mathrm{L}$
$3 \mathrm{~g} / \mathrm{L}+2 \mathrm{~g} / \mathrm{L}$
$1 \mathrm{mg} / \mathrm{L}+1 \mathrm{~g} / \mathrm{L}$
$5 \mathrm{mg} / \mathrm{L}+120 \mathrm{mg} / \mathrm{L}$
$1 \mathrm{~g} / \mathrm{L}+5 \mathrm{mg} / \mathrm{L}+120 \mathrm{mg} / \mathrm{L}$
$3 \mathrm{~g} / \mathrm{L}+1 \mathrm{mg} / \mathrm{L}+1 \mathrm{~g} / \mathrm{L}+5 \mathrm{mg} / \mathrm{L}+120 \mathrm{mg} / \mathrm{L}$
$2 \mathrm{~g} / \mathrm{L}+1 \mathrm{mg} / \mathrm{L}+1 \mathrm{~g} / \mathrm{L}+5 \mathrm{mg} / \mathrm{L}+120 \mathrm{mg} / \mathrm{L}$

preheated to $75^{\circ} \mathrm{C}$. Ethanol $(5 \mathrm{ml})$ was added, shaken, and centrifuged at $4,000 \mathrm{rpm}$ for $5 \mathrm{~min}$ to obtain the supernatant, which was fixed to $10 \mathrm{ml}$ with ethanol. The absorbance values were examined by UV spectrophotometry (Ni et al., 2005) at a wavelength of $474 \mathrm{~nm}$. An astaxanthin standard curve was constructed, and the astaxanthin content was calculated according to the standard curve equation.

\section{Fluorescence Quantitative PCR, Fatty Acid and Isopentenyl Pyrophosphate Determination}

The $120 \mathrm{~h}$ fermentation broth was taken and $18 \mathrm{~s}$ was used as the internal reference gene to perform fluorescent quantitative PCR. Quantitative PCR instrument ABI7300 and fluorescent quantitative PCR kit were used to carry out relative quantification of target gene.

The method of Li et al. (2018) was referred to determine fatty acid content. The method of Schaefer et al. (2021) was used to determine isopentenyl pyrophosphate content.

\section{Data Processing}

The experimental data in this article had at least three biological repetitions. Sigma Plot version 14.0 (Systat Software Inc., San Jose, California, United States) was used to analyze the data and make charts. SPSS version 20 (International Business Machines Corporation, Armonk, New York State, United States) was used in variance analysis. PPT version 16051 (Microsoft, Redmond, Washington, United States) and ChemDraw version 19 (PerkinElmer, Inc., Waltham, Massachusetts, United States) were used to draw pictures.

\section{RESULTS AND DISCUSSION}

\section{Effects of Single Metabolic Regulators on $P$. rhodozyma Biomass}

The biomass of $P$. rhodozyma is closely regulated to the accumulation of metabolites, such as 2-ketoglutarate and glyoxylic acid (Wang, B. et al., 2019). Glutamic acid, as a nitrogen source, promotes cell growth (Albers et al., 1996). 

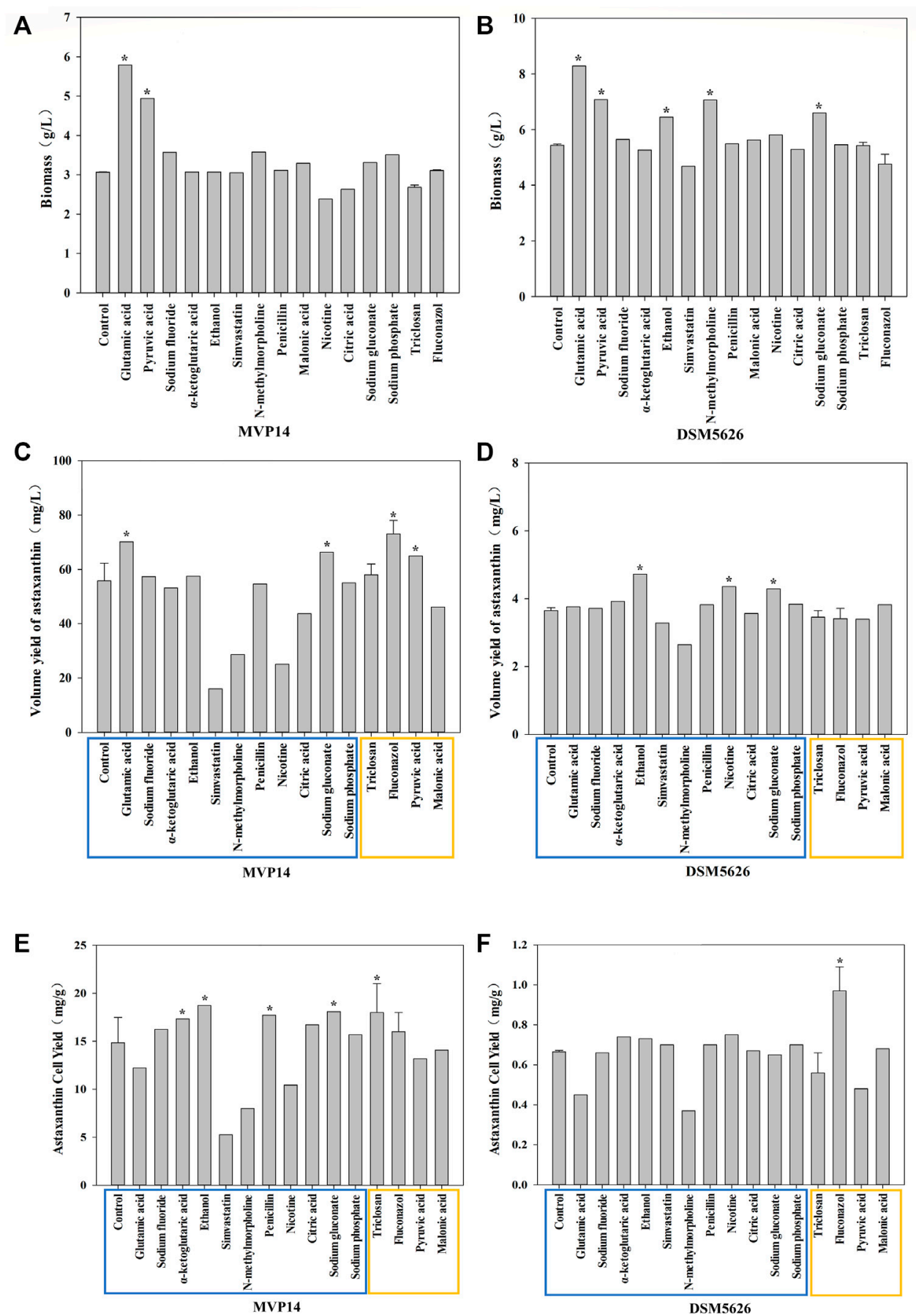

FIGURE 1 | The effect of single regulator on biomass of $P$. rhodozyma MVP14 (A) and DSM5626 (B). The effect of single regulators on astaxanthin volume yield of $P$. rhodozyma MVP14 (C) and DSM5626 (D). The effect of single regulators on astaxanthin cell yield of $P$. rhodozyma MVP14 (E) and DSM5626 (F). The blue box shows the additives acting on the synthetic pathway, and the yellow box shows the additives acting on the competitive pathway. ( ${ }^{*}$ significantly different from control, $\left.p<0.05\right)$.

Thus, the addition of glutamic acid can increase the biomass of $P$. rhodozyma. Pyruvate is an important node biomolecule for acetyl-coenzyme A (CoA) that can directly increase the substrate required for growth and thus increase biomass ( $\mathrm{Lu}$ et al., 2019). The effects of single metabolic regulators on the biomass of P. rhodozyma MVP14 and DSM5626 are shown in
Figure 1. Glutamic acid was the most prominent among the tested regulators in increasing the biomass of $P$. rhodozyma MVP14 and DSM5626, by 89 and 52.4\%, respectively. In addition, pyruvic acid remarkably increased the biomass of $P$. rhodozyma MVP14 and DSM5626 by 61 and 30.1\%, respectively. The other additives did not have a remarkable effect on 
P. rhodozyma biomass. These results are consistent with the findings of previous studies (Lan et al., 2002; Lu et al., 2019). Therefore, EMP and HMP were found to be positively correlated with $P$. rhodozyma biomass.

\section{Effects of Single Metabolic Regulators on the Volumetric Yield of Astaxanthin}

Four additives, fluconazol, glutamic acid, sodium gluconate, and pyruvic acid, significantly increased the volume yield of astaxanthin in P. rhodozyma MVP14 by 30.9, 25.8, 18.9, and $16.4 \%$, respectively, while three additives, ethanol, nicotine, and sodium gluconate, significantly increased the volume yield of astaxanthin in P. rhodozyma DSM5626 by $29.3,19.5$, and $17.5 \%$, respectively. Fluconazol has been reported to inhibit the ergosterol synthesis pathway (Yang et al., 2018), which is a competitive pathway for astaxanthin synthesis (Miao et al., 2011). Some studies have reported that adding lowconcentration ethanol can increase $\beta$-hydroxy- $\beta$ methylglutaryl-CoA reductase (HMGR) activity and promote astaxanthin synthesis (Gu et al., 1997; Xiao et al., 2015), which could explain the observed increase in volume yield of astaxanthin. The FAS pathway is a competitive pathway for pigment synthesis. When this pathway is weakened, carbon flow is increased to promote pigment synthesis and astaxanthin production (Miao et al., 2011). Astaxanthin volume yield can be increased by improving biomass and inhibiting competition pathways, such as the ergosterol synthetic and FAS pathways (Cheng et al., 2016; Yang et al., 2018). The astaxanthin volumetric yields of $P$. rhodozyma MVP14 and DSM5626 are plotted in Figure 1. Glutamic acid and fluconazol improved the volume yield of astaxanthin in the high-yield strain MVP14, by 25.8 and 30.9\%, respectively (Figure 1C). The increase in volume yield of astaxanthin by glutamic acid might be due to the effect of glutamic acid on cell biomass (Figure 1A). Meanwhile, fluconazol increased the volume yield of astaxanthin by increasing the carbon flux of the MVA pathway involved in astaxanthin synthesis. The volumetric yields in MVP14 and DSM5626 increased by 3 and $29.3 \%$, respectively, with the addition of ethanol. Moreover, triclosan, an inhibitor of FAS, also increased the astaxanthin volume yield in MVP14 by 4\%, but did not have an effect in DSM5626.

Ethanol and nicotine were the most effective in increasing the astaxanthin yield in DSM5626 (Figure 2), while glutamic acid and fluconazol were the most effective in increasing the astaxanthin yield in MVP14 (70.15 and $73 \mathrm{mg} / \mathrm{L}$, respectively) (Figure 2). Among the regulators which inhibit the competition pathways, fluconazol was the most effective and increased the yield by $30.9 \%$ in MVP14. Whereas, glutamic acid was the most effective regulator that promoted the synthetic pathways and increased the volume yield by $25.8 \%$ in MVP14. These results are consistent with the findings of previous studies (Gu et al., 1997; Miao et al., 2011; Xiao et al., 2015; Cheng et al., 2016; Yang et al., 2018). Therefore, inhibition of the FAS and ergosterol synthesis pathways, and the promotion of HMGR contributed to the enhancement of astaxanthin yield.

\section{Effects of Single Metabolic Regulators on the Astaxanthin Cell Yield of $\boldsymbol{P}$. rhodozyma}

Astaxanthin cell yield, also known as astaxanthin content, refers to the astaxanthin production capacity of a single cell. Ethanol can promote the enzymatic activity of HMGR (Gu et al., 1997), which is an essential enzyme in the MVA pathway for astaxanthin synthesis (Xiao et al., 2015). Sodium gluconate can enhance EMP (Wushensky et al., 2018), which can provide the substrate acetyl-CoA to promote astaxanthin synthesis and increase astaxanthin cell yield. Improving astaxanthin cell yield is the primary focus in industrial production of astaxanthin. The addition of ethanol, sodium gluconate, and triclosan increased the astaxanthin cell yield in P. rhodozyma MVP14 by $26.1,21.8$, and $21.2 \%$, respectively (Figure 1). In addition, nicotine can also increase the astaxanthin content in P. rhodozyma DSM5626 by $12.8 \%$ (Figure 1). These results further indicated that the inhibition of competition pathways, such as the FAS pathway, had a remarkable effect on astaxanthin synthesis (Cheng et al., 2016). The addition of these three additives had the most remarkable effect on astaxanthin production in $P$. rhodozyma. These results are consistent with the findings of previous studies (Gu et al., 1997; Xiao et al., 2015). The promotion of the EMP and MVA pathways contributed to the improvement in astaxanthin cellular yield.

The effect of a single regulator of astaxanthin cell yield on the metabolic pathway in P. rhodozyma MVP14 is shown in Figure 2. The HMP pathway is the initial pathway in astaxanthin biosynthesis that initiates conversion of glucose to astaxanthin (Pan et al., 2020). Glutamic acid promoted the HMP pathway, but reduced the astaxanthin cell yield by $18.7 \%$, as glutamic acid promotes the use of carbon sources for biomass growth and NADPH synthesis (An, 2001). Sodium phosphate can inhibit the HMP pathway, but can also promote the conversion of glucose to EMP (Liu et al., 2022); therefore, it can increase astaxanthin production by $5.6 \%$. The addition of citric acid increased astaxanthin cell yield by $12.5 \%$, as citric acid inhibits the EMP pathway, provides raw materials for the TCA cycle, and reduces the entry of acetyl-CoA into TCA cycle (Li et al., 2012).

After completion of the EMP pathway, the metabolites entered the MVA pathway. Ethanol and penicillin promoted MVA synthesis and improved astaxanthin cell yield by 26 and $19.3 \%$, respectively, whereas simvastatin and N-methyl morpholine inhibited MVA synthesis and decreased astaxanthin cell yield by 64.6 and $46.2 \%$, respectively. The FAS and ergosterol synthesis pathways are the competing pathways for astaxanthin synthesis (Pan et al., 2020). Fluconazol inhibited the ergosterol pathway and increased astaxanthin production by $7.7 \%$. Triclosan increased astaxanthin production by $21.2 \%$ by inhibiting the FAS pathway.

Ethanol and triclosan showed the best results among the promoters of the synthesis pathways and inhibitors of competition pathways, respectively. This result suggests that inhibition of the FAS pathway is of great importance for regulation of competing pathways. The improvement of 


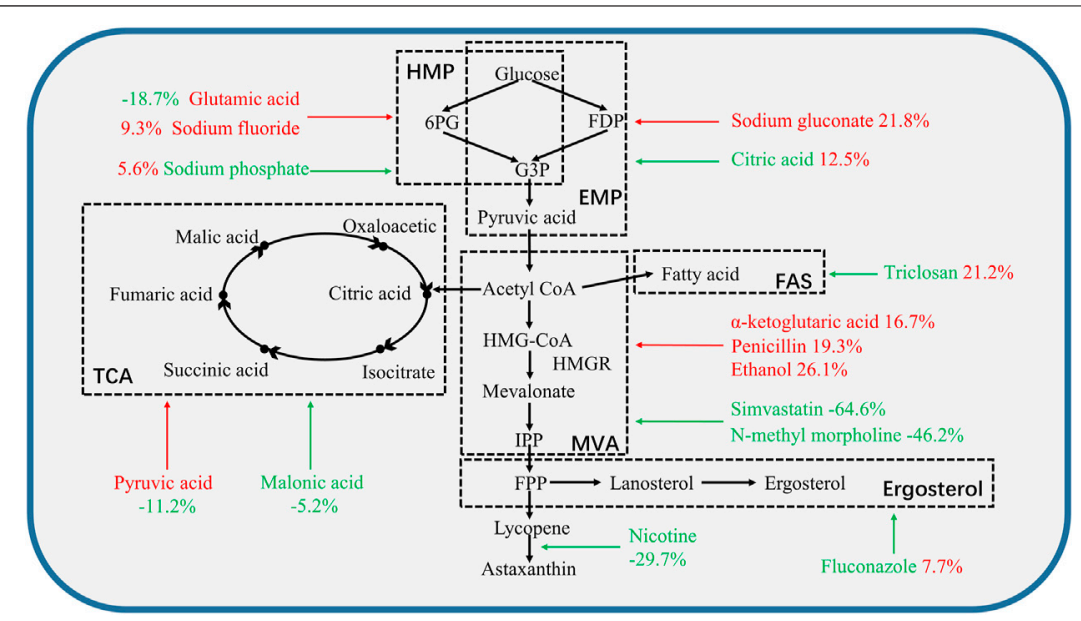

FIGURE 2 | The effect of single regulator on the metabolic pathway in P. rhodozyma MVP14. The black dashed lines frame the corresponding metabolic pathways described in black bold text. The red additives represent the promoters of this pathway or enzyme. Green additives represent inhibitors of this pathway or enzyme. Red numbers mean an increase in astaxanthin cell yield. Green numbers mean reduction of astaxanthin content. The arrow points to the pathway or site affected by the corresponding additive. HMP: hexose monophosphate pathway, EMP: Embden-Meyerhof-Parnas pathway, TCA: tricarboxylic acid cycle, MVA: mevalonate pathway, FAS: fatty acids pathway, 6PG: 6-phosphogluconate, FDP: fructose-1, 6-diphosphate, G3P: glucose-3-phosphate, IPP: isopentenyl pyrophosphate, FPP: farnesyl pyrophosphate.

astaxanthin cell yield by a single regulatory agent was limited up to $5.6-26.1 \%$, which could still be improved.

\section{Effects of Different Regulator Combinations on the Astaxanthin Production of $P$. rhodozyma}

Combinatorial regulation is a beneficial modification strategy that simultaneously targets multiple metabolic nodes and has been successfully applied in other microorganisms (Li et al., 2012; Chaturvedi et al., 2021). When metabolic regulators are combined in the fermentation process, multiple nodes of the synthesis process can be simultaneously regulated to increase the regulation efficiency. P. rhodozyma JMU-MVP14, an astaxanthin overproducing strain, was selected to further optimise production using combinations of the regulators. Based on the different effects of the single metabolic regulators, glutamate, pyruvate, sodium gluconate, citric acid, penicillin, ethanol, triclosan, and fluconazol were selected for the combination treatments, as shown in Figure 3.

Glutamic acid with penicillin, pyruvic acid with penicillin, glutamic acid with citric acid, and glutamic acid with triclosan increased the astaxanthin volume yield by $42.8,42,40.6$, and $39.2 \%$, respectively. The effect of the combination treatments was higher than the effect of the individual regulators. This suggests that their promotive effects were synergistically enhanced. Biomass and astaxanthin volume yields were increased by different combinations of glutamic acid or pyruvic acid compared to the control group. Glutamic acid was more effective than pyruvic acid in promoting the growth of the JMU-MVP14 strain, which is consistent with the results of the single regulators. The combination of glutamic acid and pyruvic acid further enhanced the growth of JMU-MVP14. This result suggests that the growth-promoting effects of the regulators can be further enhanced when the regulators are used in combination. In addition, the combination of glutamic acid and sodium gluconate had an obvious synergistic effect in promoting biomass growth and astaxanthin volumetric yield. Triclosan with fluconazol and penicillin with ethanol increased astaxanthin cell yield by 25 and $15.7 \%$, respectively. The combination of penicillin, ethanol, triclosan, and fluconazol remarkably increased astaxanthin cell yield by $51 \%$, reaching $22 \mathrm{mg} / \mathrm{g}$. These results suggest that the combined effect of the four additives on cell yield was better than that of the combination of two additives. This showed that there was still the possibility of synergy and overlap between the combined additives.

We compared the effects of the combination with the highest astaxanthin volumetric yield with that of the combination with the highest astaxanthin cell yield. The metabolic nodes affected by the combined additives were mapped to understand how the combined additives affected the metabolic pathway (Figure 4). As shown in Figure 4A, glutamic acid promoted glyceraldehyde-3phosphate synthesis, while penicillin promoted isopentenyl pyrophosphate synthesis. Both compounds are intermediate products of astaxanthin biosynthesis. Therefore, glutamic acid and penicillin simultaneously promoted astaxanthin synthesis by increasing volumetric yield. However, this combination also decreased astaxanthin cell yield as it enabled the use of a partial flux to increase biomass.

As shown in Figure 4B, penicillin with ethanol had a better regulatory effect and synergistic promotion effect than either penicillin or ethanol alone. Penicillin and ethanol have been reported to promote HMGR and MVA kinase in the 


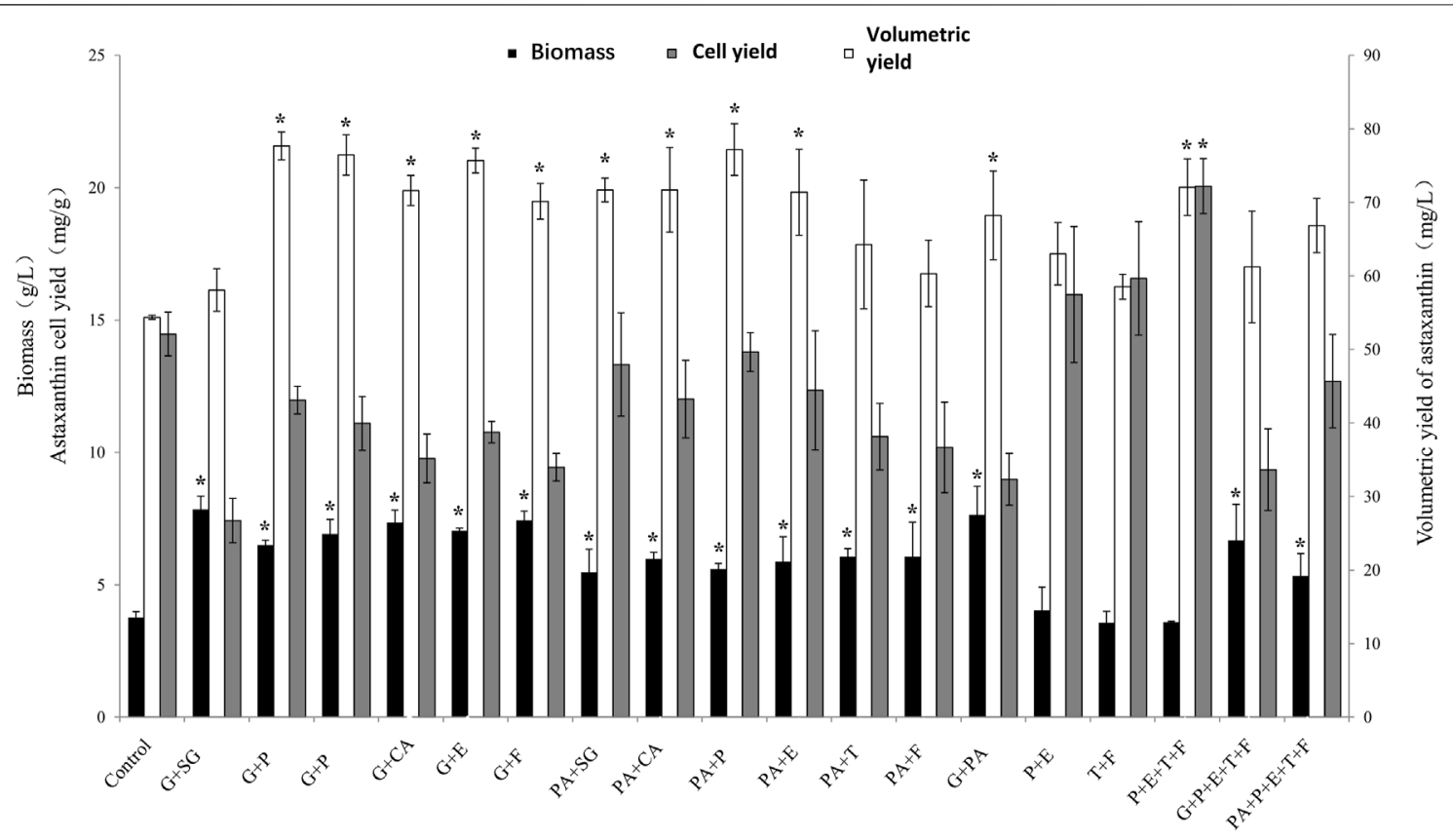

FIGURE 3 | The effect of different regulator combinations on biomass, astaxanthin cell yield and volumetric yield in $P$. rhodozyma MVP14. The abbreviation of abscissa in the figure is the following meaning, G: Glutamic acid, SG: Sodium gluconate, CA: Citric acid, P: Penicillin, E: ethanol, T: Triclosan, F: Fluconazol, PA: Pyruvic acid. (* significantly different from control, $p<0.05$ ).

astaxanthin synthetic pathway (Gu et al., 1997; Wang et al., 2012). Untreated MVP14 was used as the control group to determine the relative expression level of HMGR gene in P. rhodozyma with penicillin, triclosan, fluconazol, and ethanol (Figure 4C). The relative expression of HMGR after addition of the combination increased by $49.0 \%$. The IPP content in the MVA pathway was also measured, and the content increased by $54.8 \%$ after the addition of the combined additives (Figure 4D). It is showed that HMGR, an important synthetic enzyme in the MVA pathway, increases carotenoid production when its expression increases (Loto et al., 2012). Ergosterol is a branch point in the MVA pathway that can affect HMGR through a negative feedback mechanism (Loto et al., 2012). Therefore, ergosterol inhibition could promote astaxanthin production by inhibiting the competing pathway and enhancing HMGR expression in the synthetic pathway. Moreover, fluconazol with triclosan can inhibit the competing pathways of astaxanthin synthesis, FAS and ergosterol synthesis pathways (Cheng et al., 2016; Sanglard et al., 1998). Compared to the control group, the total fatty acid content of $P$. rhodozyma decreased by $19.3 \%$ after inhibition of the FAS pathway (Figure 4E). In general, fungi possess a very active acetyl-CoA metabolism; carotenoids and fatty acids require acetyl-CoA as a synthetic precursor (Sandmann et al., 2021). Fatty acid accumulation is 1000 -fold higher than that of carotenoids in P. rhodozyma (Miao et al., 2010), which indicates that most acetyl-CoA is used for lipid synthesis. The inhibition of the FAS pathway is therefore expected to contribute considerably to the astaxanthin synthesis pathway, including the MVA pathway. The astaxanthin content of both high- and low- yield strains increased with the addition of fluconazol and inhibition of FAS. The competing pathways were inhibited, and the synthetic pathway was enhanced; the synergistic effect in the two directions promoted astaxanthin synthesis. This combination increased both astaxanthin cell yield and volumetric yield. A comparison of Figures 1, 4A revealed that merely promoting and modulating the master pathway is not sufficient to improve astaxanthin cell yield. The promotion of master pathways should be combined with the inhibition of competing pathways to effectively increase astaxanthin cell yield. As shown in Figure 3, the combination of some regulators can increase the biomass but decrease the astaxanthin cell yield. Therefore, we speculated that if further modulation of the cell yield is necessary, a regulator that stimulates biomass growth should not be used in the combination. Therefore, when increasing cell yield, the stimulation of biomass growth should be avoided, and regulation of the main synthesis and competition pathways of astaxanthin should be the focus. This study provides theoretical support for subsequent research on genetic pathway modifications.

\section{CONCLUSION}

Fifteen single regulators of six metabolic pathways were screened to improve astaxanthin production in P. rhodozyma MVP14 and DSM5626. Glutamic acid and fluconazol were the most effective additives at improving the astaxanthin volume production in 

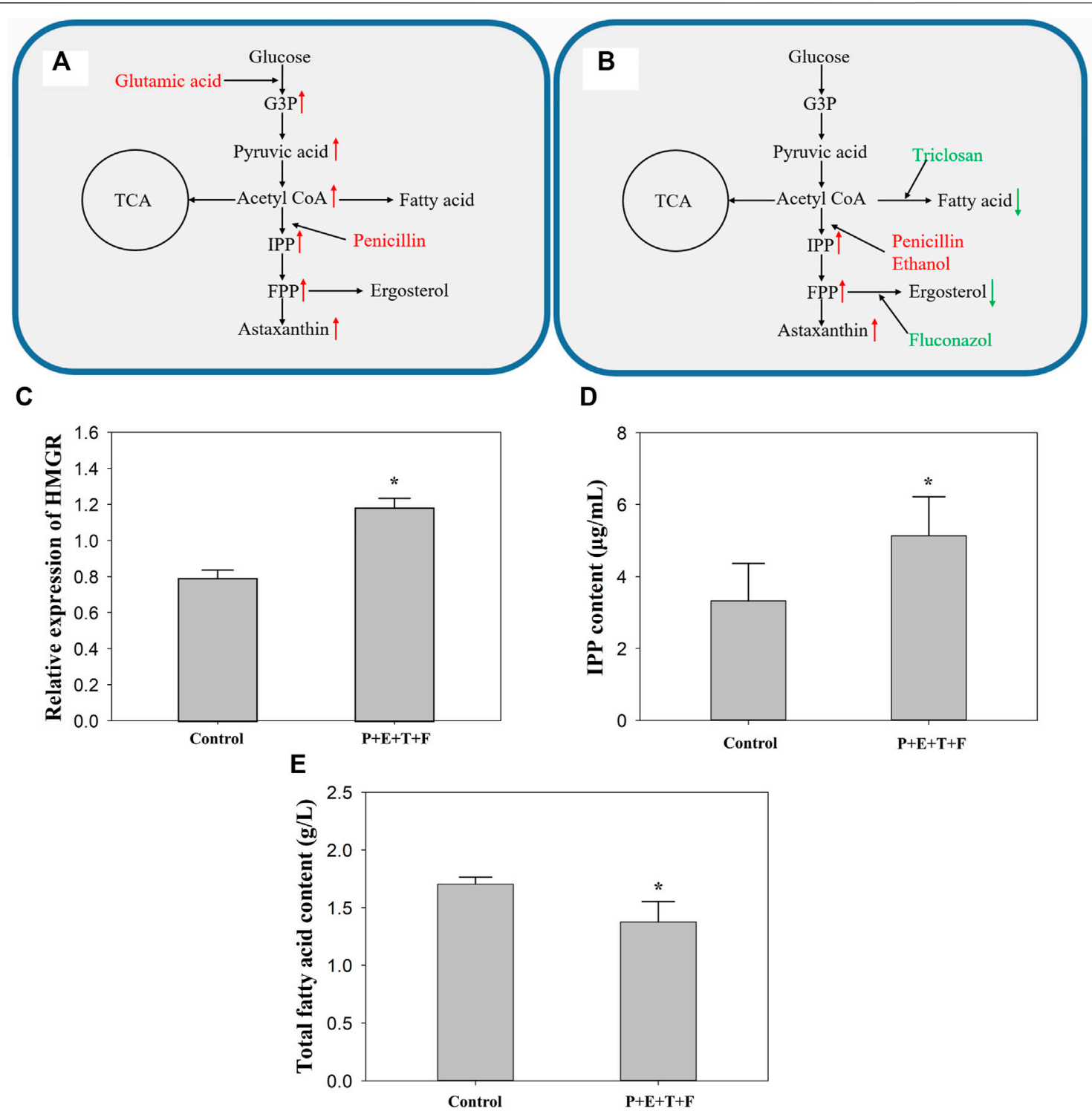

FIGURE 4 | The effect of different regulator combinations on the metabolic pathway in P. rhodozyma MVP14. (A) Highest astaxanthin volumetric yield with adding glutamic acid and penicillin. (B) Highest astaxanthin cell yield with adding penicillin, ethanol, triclosan and fluconazol. Red substance represents the promotion of current pathway, while green substance represents the inhibition. Changes in the relative expression of HMGR gene (C), IPP content (D) and total fatty acids (E) after adding penicillin, ethanol, triclosan and fluconazol to $P$. rhodozyma MVP14. The red arrow indicates that the metabolite is increased, and the green one indicates that it is decreased. G3P: glucose-3-phosphate, IPP: isopentenyl pyrophosphate, FPP: farnesyl pyrophosphate, TCA: tricarboxylic acid cycle, P: penicillin, E: ethanol, T: triclosan, F: fluconazol. (" significantly different from control, $p<0.05$ ).

MVP14, improving volumetric yield by 30.9 and $25.8 \%$, respectively. Ethanol was the most effective in increasing astaxanthin production in DSM626, by $29.3 \%$. Six effective additives were screened as combination additives. We found that although glutamic acid and penicillin simultaneously promoted the astaxanthin synthesis pathway for volumetric yield, the combination caused a decrease in astaxanthin production. The optimal combination to increase the cell yield of astaxanthin was penicillin, ethanol, triclosan, and fluconazol, with an increase of $51 \%$, reaching $22.4 \mathrm{mg} / \mathrm{g}$. In this combination, triclosan and fluconazol inhibits the competitive FAS and ergosterol pathways to increase astaxanthin cell yield. This was in synergy with the promotion of HMGR and MVA in the astaxanthin synthetic pathway by penicillin and ethanol. Our results suggest that promotion of master pathways should be combined with inhibition of competing pathways to effectively increase astaxanthin cell yield. In addition, stimulation of the biomass pathway should be avoided. This study provides theoretical support for subsequent research on the genetic modification of the astaxanthin synthesis pathway. 


\section{DATA AVAILABILITY STATEMENT}

The original contributions presented in the study are included in the article/Supplementary Material, further inquiries can be directed to the corresponding author.

\section{AUTHOR CONTRIBUTIONS}

ZL, LYa, and YZ sorted data, ZL and HY wrote original draft, $\mathrm{CZ}$ completed experiment, XD, HN, LYo,

\section{REFERENCES}

Albers, E., Larsson, C., Lidén, G., Niklasson, C., and Gustafsson, L. (1996). Influence of the Nitrogen Source on Saccharomyces cerevisiae Anaerobic Growth and Product Formation. Appl. Environ. Microbiol. 62, 3187-3195. doi:10.1128/aem.62.9.3187-3195.1996

An, G. H. (2001). Improved Growth of the Red Yeast, Phaffia Rhodozyma (Xanthophyllomyces Dendrorhous), in the Presence of Tricarboxylic Acid Cycle Intermediates. Biotechnol. Lett. 23, 1005-1009. doi:10.1023/A: 1010523800650

Batghare, A. H., Singh, N., and Moholkar, V. S. (2018). Investigations in Ultrasound-Induced Enhancement of Astaxanthin Production by Wild Strain Phaffia Rhodozyma MTCC 7536. Bioresour. Tech. 254, 166-173. doi:10.1016/j.biortech.2018.01.073

Bhosale, P. (2004). Environmental and Cultural Stimulants in the Production of Carotenoids from Microorganisms. Appl. Microbiol. Biotechnol. 63, 351-361. doi:10.1007/s00253-003-1441-1

Chaturvedi, S., Gupta, A. K., Bhattacharya, A., Dutta, T., Nain, L., and Khare, S. K. (2021). Overexpression and Repression of Key Rate-limiting Enzymes (Acetyl CoA Carboxylase and HMG Reductase) to Enhance Fatty Acid Production from Rhodotorula Mucilaginosa. J. Basic Microbiol. 61, 4-14. doi:10.1002/ jobm.202000407

Chen, J.-H., Wei, D., Lim, P.-E., Xie, J., and Chen, W. N. (2021). Screening and Effect Evaluation of Chemical Inducers for Enhancing Astaxanthin and Lipid Production in Mixotrophic Chromochloris zofingiensis. J. Appl. Phycol. doi:10.1007/s10811-021-02618-6

Cheng, Y.-r., Sun, Z.-j., Cui, G.-Z., Song, X., and Cui, Q. (2016). A New Strategy for Strain Improvement of Aurantiochytrium Sp. Based on Heavy-Ions Mutagenesis and Synergistic Effects of Cold Stress and Inhibitors of EnoylACP Reductase. Enzyme Microb. Tech. 93-94, 182-190. doi:10.1016/ j.enzmictec.2016.08.019

Domínguez-Bocanegra, A. R., Ponce-Noyola, T., and Torres-Muñoz, J. A. (2007). Astaxanthin Production by Phaffia Rhodozyma and Haematococcus pluvialis: A Comparative Study. Appl. Microbiol. Biotechnol. 75, 783-791. doi:10.1007/ s00253-007-0889-9

Fazeli, M. R., Tofighi, H., Madadkar-Sobhani, A., Shahverdi, A. R., Nejad-Sattari, T., Mirzaie, S., et al. (2009). Nicotine Inhibition of Lycopene Cyclase Enhances Accumulation of Carotenoid Intermediates byDunaliella salinaCCAP 19/18. Eur. J. Phycol. 44, 215-220. doi:10.1080/09670260802578526

Gu, W.-L., An, G.-H., and Johnson, E. A. (1997). Ethanol Increases Carotenoid Production in Phaffia Rhodozyma. J. Ind. Microbiol. Biotechnol. 19, 114-117. doi:10.1038/sj.jim.2900425

GumiŃska, M., and Sterkowicz, J. (1975). Biochemical Changes in the Blood of Humans Chronically Exposed to Fluorides. Acta. medica. Polona. 16, 215-224.

Kothari, D., Lee, J.-H., Chon, J.-W., Seo, K.-H., and Kim, S.-K. (2019). Improved Astaxanthin Production by Xanthophyllomyces Dendrorhous SK984 with Oak Leaf Extract and Inorganic Phosphate Supplementation. Food Sci. Biotechnol. 28, 1171-1176. doi:10.1007/s10068-019-00604-w

Lai, H., Liu, N., Zeng, L., Zhang, Y.-M., and Pan, W. (2021). Astaxanthin Alleviates Pathological Brain Aging through the Upregulation of Hippocampal Synaptic Proteins. Neural Regen. Res. 16, 1062-1067. doi:10.4103/1673-5374.300460 and $\mathrm{NH}$ investigated and provided advice, LL provided funding.

\section{FUNDING}

This work was financially supported by National Natural Science Foundation of China (No. 32001672, 22038012), Natural Science Foundation of Fujian Province (No. 2020J01678, 2020NZ012015) and the Scientific Research Foundation of Jimei University, China (No. 4411/(619052).

Lan, W.-Z., Qin, W.-M., and Yu, L.-J. (2002). Effect of Glutamate on Arachidonic Acid Production from Mortierella Alpina. Lett. Appl. Microbiol. 35, 357-360. doi:10.1046/j.1472-765X.2002.01195.X

Lee, K.-M., Kim, S.-K., Lee, Y.-G., Park, K.-H., and Seo, J.-H. (2018). Elimination of Biosynthetic Pathways for L-Valine and L-Isoleucine in Mitochondria Enhances Isobutanol Production in Engineered Saccharomyces cerevisiae. Bioresour. Tech. 268, 271-277. doi:10.1016/j.biortech.2018.07.150

Li, Y., Tian, C., Tian, H., Zhang, J., He, X., Ping, W., et al. (2012). Improvement of Bacterial Cellulose Production by Manipulating the Metabolic Pathways in Which Ethanol and Sodium Citrate Involved. Appl. Microbiol. Biotechnol. 96, 1479-1487. doi:10.1007/s00253-012-4242-6

Li, Z., Chen, X., Li, J., Meng, T., Wang, L., Chen, Z., et al. (2018). Functions of PKS Genes in Lipid Synthesis of Schizochytrium Sp. By Gene Disruption and Metabolomics Analysis. Mar. Biotechnol. 20, 792-802. doi:10.1007/s10126018-9849-x

Liu, C., Hu, B., Cheng, Y., Guo, Y., Yao, W., and Qian, H. (2021). Carotenoids from Fungi and Microalgae: A Review on Their Recent Production, Extraction, and Developments. Bioresour. Tech. 337, 125398. doi:10.1016/ j.biortech.2021.125398

Liu, Y. X., Jiang, X. L., Xu, Y. N., Piao, X. C., and Lian, M. L. (2022). Antibacterial Mechanisms of Orostachys Cartilaginous Cell Cultures: Effect on Cell Permeability and Respiratory Metabolism of Bacillus Subtilis. Plant Cel. Tiss. Organ. Cult. doi:10.1007/s11240-021-02160-w

Loto, I., Gutiérrez, M. S., Barahona, S., Sepúlveda, D., Martínez-Moya, P., Baeza, M., et al. (2012). Enhancement of Carotenoid Production by Disrupting the C22-Sterol Desaturase Gene (CYP61) in Xanthophyllomyces Dendrorhous. BMC Microbiol. 12, 235. doi:10.1186/1471-2180-12-235

Lu, H., Cheng, J., Zhu, Y., Li, K., Tian, J., and Zhou, J. (2019). Responses of Arthrospira ZJU9000 to High Bicarbonate Concentration (HCO3-: 171.2 mM): How Do Biomass Productivity and Lipid Content Simultaneously Increase? Algal Res. 41, 101531. doi:10.1016/j.algal.2019.101531

Miao, L., Wang, Y., Chi, S., Yan, J., Guan, G., Hui, B., et al. (2010). Reduction of Fatty Acid Flux Results in Enhancement of Astaxanthin Synthesis in a Mutant Strain of Phaffia Rhodozyma. J. Ind. Microbiol. Biotechnol. 37, 595-602. doi:10.1007/s10295-010-0706-9

Miao, L., Chi, S., Tang, Y., Su, Z., Yin, T., Guan, G., et al. (2011). Astaxanthin Biosynthesis Is Enhanced by High Carotenogenic Gene Expression and Decrease of Fatty Acids and Ergosterol in a Phaffia Rhodozyma Mutant Strain. FEMS Yeast Res. 11, 192-201. doi:10.1111/j.1567-1364.2010.00705.x

Mimoun-Benarroch, M., Lallement, J., Rhazi, L., Boroch, C., Hugot, C., Niamba, C.-N., et al. (2018). Free Form Astaxanthin from Yeast Phaffia Rhodozyma Fermentation Reduces Plasmatic Triglycerides in a Pre-Obesity Diet-Induced Dyslipidaemia Mouse Model. J. Food Compost. Anal. 65, 11-15. doi:10.1016/ j.jfca.2017.07.023

Ni, H., He, G.-q., Ruan, H., Chen, Q.-h., and Chen, F. (2005). Application of Derivative Ratio Spectrophotometry for Determination of $\beta$-Carotene and Astaxanthin fromPhaffia Rhodozymaextract. J. Zhejiang Univ. Sci. 6B, 514-522. doi:10.1631/jzus.2005.B0514

Ni, H., Chen, Q.-h., He, G.-q., Wu, G.-b., and Yang, Y.-f. (2008). Optimization of Acidic Extraction of Astaxanthin from Phaffia Rhodozyma. J. Zhejiang Univ. Sci. B 9, 51-59. doi:10.1631/jzus.B061261

Pan, X., Wang, B., Duan, R., Jia, J., Li, J., Xiong, W., et al. (2020). Enhancing Astaxanthin Accumulation in Xanthophyllomyces Dendrorhous by a 
Phytohormone: Metabolomic and Gene Expression Profiles. Microb. Biotechnol. 13, 1446-1460. doi:10.1111/1751-7915.13567

Sandmann, G., Pollmann, H., Gassel, S., and Breitenbach, J. (2021). "Xanthophyllomyces Dendrorhous, a Versatile Platform for the Production of Carotenoids and Other Acetyl-CoA-Derived Compounds," in Carotenoids: Biosynthetic and Biofunctional Approaches, Advances in Experimental Medicine and Biology. Editor N. Misawa (Singapore: Springer), 1261, 137-151. doi:10.1007/978-981-15-7360-6_11

Sanglard, D., Ischer, F., Koymans, L., and Bille, J. (1998). Amino Acid Substitutions in the Cytochrome P-450 Lanosterol 14 $\alpha$-Demethylase (CYP51A1) from AzoleResistant Candida Albicans Clinical Isolates Contribute to Resistance to Azole Antifungal Agents. Antimicrob. Agents Chemother. 42, 241-253. doi:10.1128/ aac.42.2.241

Schaefer, V. D., Müller, V. V., Feltraco Lizot, L. d. L., Hahn, R. Z., Schneider, A., Antunes, M. V., et al. (2021). Sensitive Determination of 11-Nor-9-Carboxy$\Delta 9$-Tetrahydrocannabinol and Complementary Cannabinoids in Hair Using Alkaline Digestion and Mixed-Mode Solid Phase Extraction Followed by Liquid-Chromatography-Tandem Mass Spectrometry. Forensic Sci. Int. 328, 111047. doi:10.1016/j.forsciint.2021.111047

Schmidt, I., Schewe, H., Gassel, S., Jin, C., Buckingham, J., Hümbelin, M., et al. (2011). Biotechnological Production of Astaxanthin with Phaffia Rhodozyma/ Xanthophyllomyces Dendrorhous. Appl. Microbiol. Biotechnol. 89, 555-571. doi:10.1007/s00253-010-2976-6

Sedmak, J. J., Weerasinghe, D. K., and Jolly, S. O. (1990). Extraction and Quantitation of Astaxanthin from Phaffia Rhodozyma. Biotechnol. Tech. 4, 107-112. doi:10.1007/BF00163282

Stoklosa, R. J., Johnston, D. B., and Nghiem, N. P. (2019). Phaffia Rhodozyma Cultivation on Structural and Non-structural Sugars from Sweet Sorghum for Astaxanthin Generation. Process Biochem. 83, 9-17. doi:10.1016/ j.procbio.2019.04.005

Sun, D., Zhang, Z., Zhang, Y., Cheng, K.-W., and Chen, F. (2019). Light Induces Carotenoids Accumulation in a Heterotrophic Docosahexaenoic Acid Producing Microalga, Crypthecodinium Sp. SUN. Bioresour. Tech. 276, 177-182. doi:10.1016/j.biortech.2018.12.093

Susilowati, R., Jannah, J., Maghfuroh, Z., and Kusuma, M. (2020). Antihyperlipidemic Effects of Apple Peel Extract in High-Fat Diet-Induced Hyperlipidemic Rats. J. Adv. Pharm. Technol. Res. 11, 128-133. doi:10.4103/ japtr.JAPTR_28_20

Torres-Haro, A., Verdín, J., Kirchmayr, M. R., and Arellano-Plaza, M. (2021). Metabolic Engineering for High Yield Synthesis of Astaxanthin in Xanthophyllomyces Dendrorhous. Microb. Cel Fact. 20, 1-17. doi:10.1186/ s12934-021-01664-6

Wan, X., Zhou, X.-R., Moncalian, G., Su, L., Chen, W.-C., Zhu, H.-Z., et al. (2021). Reprogramming Microorganisms for the Biosynthesis of Astaxanthin via Metabolic Engineering. Prog. Lipid Res. 81, 101083. doi:10.1016/j.plipres.2020.101083

Wang, B., Pan, X., Jia, J., Xiong, W., Manirafasha, E., Ling, X., et al. (2019). Strategy and Regulatory Mechanisms of Glutamate Feeding to Enhance Astaxanthin
Yield in Xanthophyllomyces Dendrorhous. Enzyme Microb. Tech. 125, 45-52. doi:10.1016/j.enzmictec.2019.02.010

Wang, J.-F., Liu, X.-J., Liu, R.-S., Li, H.-M., and Tang, Y.-J. (2012). Optimization of the Mated Fermentation Process for the Production of Lycopene by Blakeslea Trispora NRRL $2895(+)$ and NRRL 2896 (-). Bioproc. Biosyst. Eng. 35, 553-564. doi:10.1007/s00449-011-0628-6

Wang, L., Zhao, Y., Ge, J., Zhu, L., Wei, Z., Wu, J., et al. (2019). Effect of Tricarboxylic Acid Cycle Regulators on the Formation of Humic Substance during Composting: The Performance in Labile and Refractory Materials. Bioresour. Tech. 292, 121949. doi:10.1016/j.biortech.2019.121949

Wushensky, J. A., Youngster, T., Mendonca, C. M., and Aristilde, L. (2018). Flux Connections between Gluconate Pathway, Glycolysis, and Pentose-Phosphate Pathway during Carbohydrate Metabolism in Bacillus Megaterium QM B1551. Front. Microbiol. 9, 1-13. doi:10.3389/fmicb.2018.02789

Xiao, A., Jiang, X., Ni, H., Yang, Q., and Cai, H. (2015). Study on the Relationship between Intracellular Metabolites and Astaxanthin Accumulation during Phaffia Rhodozyma Fermentation. Electron. J. Biotechnol. 18, 148-153. doi:10.1016/j.ejbt.2015.02.002

Yang, Z., Wang, Q., Ma, K., Shi, P., Liu, W., and Huang, Z. (2018). Fluconazole Inhibits Cellular Ergosterol Synthesis to Confer Synergism with Berberine against Yeast Cells. J. Glob. Antimicrob. Resist. 13, 125-130. doi:10.1016/j.jgar.2017.12.011

Zhang, J., Li, Q., Lu, Y., Guan, X., Liu, J., Xu, N., et al. (2021). Astaxanthin Overproduction of Phaffia Rhodozyma PR106 under Titanium Dioxide Stress by Transcriptomics and Metabolic Regulation Analysis. Bioresour. Tech. 342, 125957. doi:10.1016/j.biortech.2021.125957

Zhou, X., Zhang, J., Li, Y., Cui, L., Wu, K., and Luo, H. (2021). Astaxanthin Inhibits Microglia M1 Activation against Inflammatory Injury Triggered by Lipopolysaccharide through Down-Regulating miR-31-5p. Life Sci. 267, 118943. doi:10.1016/j.lfs.2020.118943

Conflict of Interest: The authors declare that the research was conducted in the absence of any commercial or financial relationships that could be construed as a potential conflict of interest.

Publisher's Note: All claims expressed in this article are solely those of the authors and do not necessarily represent those of their affiliated organizations, or those of the publisher, the editors and the reviewers. Any product that may be evaluated in this article, or claim that may be made by its manufacturer, is not guaranteed or endorsed by the publisher.

Copyright $\odot 2022 \mathrm{Li}$, Yang, Zheng, Du, Ni, He, Yang, You, Zhu and Li. This is an open-access article distributed under the terms of the Creative Commons Attribution License (CC BY). The use, distribution or reproduction in other forums is permitted, provided the original author(s) and the copyright owner(s) are credited and that the original publication in this journal is cited, in accordance with accepted academic practice. No use, distribution or reproduction is permitted which does not comply with these terms. 